\title{
Correction to: Coronary Artery Calcium: Recommendations for Risk Assessment in Cardiovascular Prevention Guidelines
}

Mahmoud Al Rifai, MD, MPH',2

Miguel Cainzos-Achirica, MD, MPH $2,3,4,5$

Sina Kianoush, $M D, M P H^{2,6}$

Mohammadhassan Mirbolouk, $M D^{2}$

Allison Peng, $B S^{2}$

Josep Comin-Colet, $M D, P h D^{3,4,7}$

Michael J. Blaha, MD, MPH ${ }^{2,8, *}$

\author{
Address \\ ${ }^{1}$ Department of Internal Medicine, The University of Kansas School of Medicine, \\ Wichita, KS, USA \\ *,2Johns Hopkins Ciccarone Center for the Prevention of Cardiovascular Disease, \\ Johns Hopkins Medical Institutions, Blalock 524D1, 600 N Wolfe St, Baltimore, \\ MD, 21287, USA \\ Email:mblaha1@jhmi.edu \\ ${ }^{3}$ Department of Cardiology, Bellvitge University Hospital, L'Hospitalet de \\ Llobregat, Barcelona, Spain \\ ${ }^{4}$ Bellvitge Biomedical Research Institute (IDIBELL), L'Hospitalet de Llobregat, \\ Barcelona, Spain \\ ${ }^{5}$ RTI Health Solutions, Pharmacoepidemiology and Risk Management, Barcelona, \\ Spain \\ ${ }^{6}$ Department of Internal Medicine, Yale New Haven Medical Center Waterbury \\ Program, Waterbury, CT, USA \\ ${ }^{7}$ Department of Clinical Sciences, School of Medicine, Universitat de Barcelona, \\ Barcelona, Spain \\ ${ }^{8}$ Department of Epidemiology, Johns Hopkins Bloomberg School of Public Health, \\ Johns Hopkins University, Baltimore, MD, USA
}

Published online: 18 0ctober 2018

(C) Springer Science+Business Media, LLC, part of Springer Nature 2018

The online version of the original article can be found at https://doi.org/10.1007/s11936-018-0685-0 


\section{Correction to: Curr Treat Options Cardio Med https://doi.org/10.1007/s11936-018-0685-0}

In the recently published review "Coronary Artery Calcium: Recommendations for Risk Assessment in Cardiovascular Prevention Guidelines," the following author name was inadvertently misspelled as Alison Peng. The correct spelling of the author's name is: Allison Peng as shown above.

The original article has been corrected. 